\title{
ON THE NONAUTONOMOUS VOLTERRA-LOTKA COMPETITION EQUATIONS
}

\author{
SHAIR AHMAD
}

(Communicated by Charles C. Pugh)

\begin{abstract}
A nonautonomous competitive Lotka-Volterra system of two equations is considered. It is shown that if the coefficients are continuous and satisfy certain inequalities, then any solution that is positive at some point has the property that one of its components vanishes while the other approaches a certain solution of the logistic equation.
\end{abstract}

\section{INTRODUCTION}

Consider the nonautonomous system of differential equations

$$
\left\{\begin{array}{l}
u^{\prime}(t)=u(t)[a(t)-b(t) u(t)-c(t) v(t)], \\
v^{\prime}(t)=v(t)[d(t)-e(t) u(t)-f(t) v(t)]
\end{array}\right.
$$

where the functions $a(t), b(t), \ldots$, and $f(t)$ are assumed to be continuous and bounded above and below by positive constants. Given a function $g(t)$, we let $g_{L}$ and $g_{M}$ denote $\inf _{-\infty<t<\infty} g(t)$ and $\sup _{-\infty<t<\infty} g(t)$, respectively. In [2] it was shown that if the inequalities

$$
a_{L} f_{L}>c_{M} d_{M} \text { and } b_{L} d_{L}>a_{M} e_{M}
$$

hold, and if the coefficients $a(t), \ldots$, and $f(t)$ are almost periodic, then $(*)$ has a unique almost periodic solution whose components are bounded above and below by positive constants, which is globally asymptotically stable. The general case, not involving almost periodicity, was considered in [1]. The periodic case was studied in [4]. A similar system, involving reaction-diffusion equations, with periodic coefficients was studied in [3]. For the ecological significance of the system $(*)$, the reader is referred to [7] and the works cited above.

The purpose of this paper is to study $(*)$ under the assumption that the inequalities

$$
a_{L} f_{L}>c_{M} d_{M} \text { and } b_{M} d_{M} \leq a_{L} e_{L}
$$

hold.

In particular, we will show that if $\operatorname{col}(u(t), v(t))$ is a solution of $(*)$ satisfying the inequalities $u\left(t_{0}\right)>0$ and $v\left(t_{0}\right)>0$ for some number $t_{0}$, then

Received by the editors May 27, 1991.

1991 Mathematics Subject Classification. Primary 34C27; Secondary 34D05.

Key words and phrases. Positive, bounded, continuous, logistic equation, uniform convergence. 
$\lim _{t \rightarrow \infty} v(t)=0$ and $\lim _{t \rightarrow \infty}\left[u(t)-u^{*}(t)\right]=0$, where $u^{*}(t)$ is the unique solution of the logistic equation

$$
u^{\prime}(t)=u(t)[a(t)-b(t) u(t)]
$$

such that $0<\delta \leq u^{*}(t) \leq \Delta<\infty$ for certain numbers $\delta$ and $\Delta$.

Of course, a similar result, where the roles of $u$ and $v$ are interchanged, will hold if the inequalities in (1) are replaced by

$$
a_{M} f_{M} \leq c_{L} d_{L} \text { and } b_{L} d_{L}>a_{M} e_{M} .
$$

This generalizes the constant coefficient case, which can be easily verified from a phase space analysis of the system (see, e.g., [7]).

We wish to point out that the main result in this paper and some of the methods used have been motived by [3]. Also, Zhou and Pao [8] established a somewhat similar result for a system of reaction-diffusion equations where the coefficients were assumed to be positive constants. Also see Gopalsamy [6].

\section{Preliminary Lemmas}

Henceforth we shall assume that the functions $a(t), b(t), \ldots$, and $f(t)$ are continuous, bounded above and below by positive constants, and satisfy the inequalities (1).

Lemma 1. Let $\operatorname{col}(u(t), v(t))$ be a solution of $(*)$ such that $u\left(t_{0}\right)>0$ and $v\left(t_{0}\right)>0$. If $u(t) \geq \varepsilon$ for all $t \geq t_{0}$, where $\varepsilon$ is a positive number, then $\lim _{t \rightarrow \infty} v(t)=0$.

Proof. It follows (see [1]) that $u(t)$ and $v(t)$ are bounded and positive for all $t, t \geq t_{0}$. Let $\underline{u}=\lim _{t \rightarrow \infty} \inf u(t)$ and $\bar{v}=\lim _{t \rightarrow \infty} \sup v(t)$. Then $\underline{u} \geq \varepsilon>0$ and $\bar{v} \geq 0$. It suffices to show that $\bar{v}=0$. Suppose that $\bar{v}>0$. In order to obtain a contradiction, we first establish the inequality

$$
a_{L} \leq b_{M} \underline{u}+c_{M} \bar{v} .
$$

We consider the following two cases:

Case 1. Suppose that $u^{\prime}(t)$ has arbitrarily large zeros. Let $\left\{s_{n}\right\}_{n=1}^{\infty}$ be a sequence of zeros of $u^{\prime}(t)$, where $s_{n} \rightarrow \infty$ as $n \rightarrow \infty$. Let $\left\{\tau_{n}\right\}_{n=1}^{\infty}$ be a sequence of numbers such that $\tau_{n} \rightarrow \infty$ and $u\left(\tau_{n}\right) \rightarrow \underline{u}$ as $n \rightarrow \infty$. We can assume that for each $n$ there exists an integer $m_{n}$ satisfying $s_{m_{n}}<\tau_{n}<s_{m_{n+1}}$. Let $\sigma_{n} \in\left[s_{m_{n}}, s_{m_{n+1}}\right]$ such that $u\left(\sigma_{n}\right)$ is the minimum of $u(t)$ on the interval $\left[s_{m_{n}}, s_{m_{n+1}}\right]$. Since $u\left(\sigma_{n}\right) \leq u\left(\tau_{n}\right)$, it follows that $\lim \sup u\left(\sigma_{n}\right) \leq \lim \inf u\left(\sigma_{n}\right)$. Hence, $\lim _{n \rightarrow \infty} u\left(\sigma_{n}\right)$ exists and $\lim _{n \rightarrow \infty} u\left(\sigma_{n}\right)=\underline{u}$. Clearly, $u^{\prime}\left(\sigma_{n}\right)=0$. Therefore, from the first equation of $(*)$, we obtain $a\left(\sigma_{n}\right)=b\left(\sigma_{n}\right) u\left(\sigma_{n}\right)+$ $c\left(\sigma_{n}\right) v\left(\sigma_{n}\right)$ and hence $a_{L} \leq b_{M} u\left(\sigma_{n}\right)+c_{M} \sup _{t \geq \sigma_{n}} v(t)$. By taking the limit of the right-hand side as $n \rightarrow \infty$, we obtain the inequality $a_{L} \leq b_{M} \underline{u}+c_{M} \bar{v}$.

Case 2. Now suppose that $u^{\prime}(t) \neq 0$ for $t \geq t_{1}$ for some number $t_{1}$. Then $\lim _{t \rightarrow \infty} u(t)$ exists and $\lim _{t \rightarrow \infty} u(t)=\underline{u}$. Since $u(t)$ is bounded, there exists a sequence $\left\{\zeta_{n}\right\}_{n=1}^{\infty}$ such that $u^{\prime}\left(\zeta_{n}\right) \rightarrow 0$ as $n \rightarrow \infty$. Hence, $a\left(\zeta_{n}\right)-$ $b\left(\zeta_{n}\right) u\left(\zeta_{n}\right)-c\left(\zeta_{n}\right) v\left(\zeta_{n}\right) \rightarrow 0$ as $n \rightarrow \infty$. Since $a_{L}-b_{M} u\left(\zeta_{n}\right)-c_{M} \sup _{t \geq \zeta_{n}} v(t) \leq$ $a\left(\zeta_{n}\right)-b\left(\zeta_{n}\right) u\left(\zeta_{n}\right)-c\left(\zeta_{n}\right) v\left(\zeta_{n}\right)$, we obtain, by taking limits, $a_{L}-b_{M} \underline{u}-c_{M} \bar{v} \leq 0$.

It follows from a similar argument that

$$
d_{M} \geq e_{L} \underline{u}+f_{L} \bar{v} .
$$


For example, in Case 1, where $v^{\prime}(t)$ would have arbitrarily large zeros, one would let $v\left(\sigma_{n}\right)$ to be the maximum of $v(t)$ on $\left[s_{m_{n}}, s_{m_{n+1}}\right]$.

Now, multiplying (4) by $-c_{M} / f_{L}$ and adding the result to (3), we obtain

$$
a_{L}-\frac{c_{M}}{f_{L}} d_{M} \leq\left[b_{M}-\frac{c_{M}}{f_{L}} e_{L}\right] \underline{u} .
$$

Thus it follows from (1) and the fact that $u \geq \varepsilon>0$ that $\left(b_{M} f_{L}-c_{M} e_{L}\right) / f_{L}$ $>0$. Similarly, multiplying (3) by $-e_{L} / b_{M}$ and adding it to (4), we obtain $\left[\left(b_{M} f_{L}-c_{M} e_{L}\right) / b_{M}\right] \bar{v} \leq 0$. Since from the above inequality we have $b_{M} f_{L}-$ $c_{M} e_{L}>0$ and $b_{M}>0$, it follows that $\bar{v} \leq 0$, which is a contradiction. This completes the proof of the lemma.

Lemma 2. Let $k$ and $\varepsilon$ be numbers such that $k>d_{M} / f_{L}, \varepsilon>0$, and $a_{L}-$ $b_{M} \varepsilon-c_{M} k>0$. If $\operatorname{col}(\hat{u}(t), \hat{v}(t))$ is a solution of $(*)$ such that $\hat{u}\left(t_{0}\right)=\varepsilon$ and $\hat{v}\left(t_{0}\right)=k$, then $\hat{v}(t) \rightarrow 0$ as $t \rightarrow \infty$.

Proof. We note that

$$
\begin{aligned}
\hat{u}^{\prime}\left(t_{0}\right) & =\hat{u}\left(t_{0}\right)\left[a\left(t_{0}\right)-b\left(t_{0}\right) \hat{u}\left(t_{0}\right)-c\left(t_{0}\right) \hat{v}\left(t_{0}\right)\right] \\
& \geq \varepsilon\left[a_{L}-b_{M} \varepsilon-c_{M} k\right]>0, \\
\hat{v}^{\prime}\left(t_{0}\right) & =\hat{v}\left(t_{0}\right)\left[d\left(t_{0}\right)-e\left(t_{0}\right) \hat{u}\left(t_{0}\right)-f\left(t_{0}\right) \hat{v}\left(t_{0}\right)\right] \\
& \leq k\left[d_{M}-f\left(t_{0}\right) k\right]<0
\end{aligned}
$$

since $k>d_{M} / f_{L}$. We wish to show that $\hat{u}(t)>\varepsilon$ and $\hat{v}(t)<k$ for all $t>t_{0}$. These inequalities certainly hold for $t$ close to $t_{0}$ and $t>t_{0}$ since $\hat{u}^{\prime}\left(t_{0}\right)>0$ and $\hat{v}^{\prime}\left(t_{0}\right)<0$. If they did not hold for all $t>t_{0}$, then there would exist a number $\bar{t}$ such that $\hat{u}(t)>\varepsilon$ and $\hat{v}(t)<k$ for $t_{0}<t<\bar{t}$ and either (a) $\hat{u}(\bar{t})=\varepsilon$ or (b) $\hat{v}(\bar{t})=k$. If (a) held, then we would have $\hat{u}(\bar{t}) \leq 0$ and $\hat{v}(\bar{t}) \leq k$. Therefore, we would have

$$
0 \geq \hat{u}^{\prime}(\bar{t})=\hat{u}(\bar{t})[a(\bar{t})-b(\bar{t}) \hat{u}(\bar{t})-c(\bar{t}) \hat{v}(\bar{t})] \geq \varepsilon\left[a_{L}-b_{M} \varepsilon-c_{M} k\right]>0,
$$

a contradiction. If (b) held, then we would have $0 \leq \hat{v}^{\prime}(\bar{t})$ and $\varepsilon \leq \hat{u}(\bar{t})$. But $\hat{v}^{\prime}(\bar{t})=k[d(\bar{t})-e(\bar{t}) \hat{u}(\bar{t})-f(\bar{t}) k]<k\left[d_{M}-f_{L} k\right]<0$, again a contradiction. The assertion of this lemma now follows from Lemma 1.

Lemma 3. There exists a unique solution $u^{*}(t)$ of the logistic equation

$$
u^{\prime}(t)=u(t)[a(t)-b(t) u(t)]
$$

such that $\delta \leq u^{*}(t) \leq \Delta$ on $(-\infty, \infty)$, where $\Delta$ and $\delta$ are any numbers satisfying the inequalities $0<\delta<a_{L} / b_{M}$ and $a_{M} / b_{L}<\Delta$.

It appears that this lemma ought to be known. In fact, the referee of this paper has pointed out that it follows from [5]. While this appears to be the case, it is not obvious. Therefore, for the convenience of the reader we give here an independent and elementary proof.

Proof. For each positive integer $n$, let $u_{n}(t)$ be the solution of (L) satisfying $u_{n}(-n)=\Delta$. Then $u_{n}^{\prime}(-n)=\Delta[a(-n)-b(-n) \Delta] \leq \Delta\left[a_{M}-b_{L} \Delta\right]<0$. Hence for $t+n$ small and positive we have $\delta<u_{n}(t)<\Delta$. It follows that this inequality holds for all $t>-n$. For, suppose not. Then there exists a number $\bar{t}>-n$, such that $\delta<u_{n}(t)<\Delta$ for $-n<t<\bar{t}$ and either (i) $u_{n}(\bar{t})=\delta$ or (ii) $u_{n}(\bar{t})=$ 
$\Delta$. In the first case we must have $u_{n}^{\prime}(\bar{t}) \leq 0$. But $u_{n}^{\prime}(\bar{t})=\delta[a(\bar{t})-b(\bar{t}) \delta]>$ $\delta\left[a_{L}-b_{M} \delta\right]>0$, a contradiction. In the second case we have $u_{n}^{\prime}(\bar{t}) \geq 0$. But $u_{n}^{\prime}(\bar{t})=\Delta[a(\bar{t})-b(\bar{t}) \Delta] \leq \Delta\left[a_{M}-b_{L} \Delta\right]<0$, again a contradiction. This shows that $\delta<u_{n}(t)<\Delta$ holds for all $t>-n$. In particular, $\delta<u_{n}(0)<\Delta$ holds for all positive integers $n$. Therefore, there exists a subsequence $\left\{u_{n_{k}}(0)\right\}_{k=1}^{\infty}$ of $\left\{u_{n}(0)\right\}$ such that $u_{n_{k}}(0) \rightarrow u_{0}$ as $k \rightarrow \infty, \delta \leq u_{0} \leq \Delta$. Let $u^{*}(t)$ be the solution of $(\mathrm{L})$ such that $u^{*}(0)=u_{0}$. Then, since each $u_{n_{k}}(t)$ satisfies $(\mathrm{L})$ and $u_{n_{k}}(0) \rightarrow u_{0}$ as $k \rightarrow \infty$, it follows that $u_{n_{k}}(t) \rightarrow u^{*}(t)$ uniformly with respect to $t$ on compact subintervals of $(-\infty, \infty)$. Since for each number $t_{1}$, $\delta<u_{n_{k}}\left(t_{1}\right)<\Delta$ if $-n_{k}<t_{1}$, we must have $\delta \leq u^{*}\left(t_{1}\right) \leq \Delta$.

In order to establish the uniqueness, we assume that $(\bar{L})$ has two solutions $U_{1}$ and $U_{2}$ satisfying $\delta \leq U_{k}(t) \leq \Delta$ for $k=1,2$ and $t \in(-\infty, \infty)$. Since we have a first-order differential equation, we can assume, by uniqueness, that $\delta<$ $U_{1}(t)<U_{2}(t)<\Delta$. Now, $(d / d t) \ln U_{1}-(d / d t) \ln U_{2}=b(t)\left(U_{2}(t)-U_{1}(t)\right)>0$. This shows that $\ln U_{1}(t) / U_{2}(t)$, and hence $U_{1}(t) / U_{2}(t)$ is strictly increasing. Thus, $U_{1}(t) / U_{2}(t)<U_{1}(0) / U_{2}(0)<1$ for $t<0$ and

$$
\begin{aligned}
\frac{d}{d t} \ln \frac{U_{1}(t)}{U_{2}(t)} & =b(t)\left[U_{2}(t)-U_{1}(t)\right] \geq b_{L} U_{2}(t)\left[1-\frac{U_{1}(t)}{U_{2}(t)}\right] \\
& \geq b_{L} \delta\left[1-\frac{U_{1}(0)}{U_{2}(0)}\right]>0 \quad \text { for } t \leq 0 .
\end{aligned}
$$

Integrating from $T$ to $0, T<0$, we obtain

$$
\begin{aligned}
\ln \frac{U_{1}(0)}{U_{2}(0)}-\ln \frac{U_{1}(T)}{U_{2}(T)} & =\int_{T}^{0} b(t)\left(U_{2}(t)-U_{1}(t)\right) d t \\
& \geq \int_{T}^{0} b_{L} \delta\left[1-\frac{U_{1}(0)}{U_{2}(0)}\right] d t>0 .
\end{aligned}
$$

Therefore,

$$
\ln \frac{U_{1}(T)}{U_{2}(T)} \leq \ln \frac{U_{1}(0)}{U_{2}(0)}-\int_{T}^{0} b_{L} \delta\left[1-\frac{U_{1}(0)}{U_{2}(0)}\right] d t .
$$

This shows that $\lim _{T \rightarrow-\infty} \ln U_{1}(T) / U_{2}(T)=-\infty$. Consequently, since $\delta \leq$ $U_{1}(t)<U_{2}(t)<\Delta$, we conclude that $U_{1}(T) / U_{2}(T) \rightarrow 0$ as $T \rightarrow-\infty$. But, $U_{1}(T) / U_{2}(T) \geq \delta / U_{2}(T) \geq \delta / \Delta$, which leads to a contradiction. This completes the proof of Lemma 3.

Lemma 4. Let $k, \varepsilon$, and $\delta$ be numbers such that $k>d_{M} / f_{L}, 0<\varepsilon<\delta<$ $a_{L} / b_{M}$, and $a_{L}-b_{M} \varepsilon-c_{M} k>0$. If $\operatorname{col}(\hat{u}(t), \hat{v}(t))$ is a solution of $(*)$ such that $\operatorname{col}\left(\hat{u}\left(t_{0}\right), \hat{v}\left(t_{0}\right)\right)=\operatorname{col}(\varepsilon, k)$, then $u^{*}(t)-\hat{u}(t) \rightarrow 0$ as $t \rightarrow \infty$, where $u^{*}(t)$ is the unique solution of Lemma 3.

Proof. As shown in the proof of Lemma 2, $\hat{u}(t) \geq \varepsilon$ for $t \geq t_{0}$. Moreover, (see [1]), $\hat{u}$ is bounded above for $t \geq t_{0}$. Let $w(t)=1 / \hat{u}(t)$ and $w^{*}(t)=1 / u^{*}(t)$ for $t \geq t_{0}$. We have

$$
\begin{aligned}
w^{\prime}(t) & =-a(t) w(t)+b(t)+c(t) \hat{v}(t) w(t), \\
w^{*^{\prime}}(t) & =-a(t) w^{*}(t)+b(t),
\end{aligned}
$$

and hence

$$
w^{\prime}(t)-w^{*^{\prime}}(t)=-a(t)\left(w(t)-w^{*}(t)\right)+c(t) \hat{v}(t) w(t)
$$

for $t \geq t_{0}$ 
We consider two possibilities:

(I) There exists $t_{1} \geq t_{0}$ such that $\left(w-w^{*}\right)^{\prime}(t) \neq 0$ for $t \geq t_{1}$.

(II) There exists a sequence of numbers $\left\{s_{n}\right\}_{1}^{\infty}$ in $\left[t_{0}, \infty\right)$ such that for $n \geq 1, s_{n}<s_{n+1},\left(w-w^{*}\right)^{\prime}\left(s_{n}\right)=0$, and $s_{n} \rightarrow \infty$ as $n \rightarrow \infty$.

If (I) holds, then $\lim _{t \rightarrow \infty}\left(w(t)-w^{*}(t)\right)$ exists. If $\lim _{t \rightarrow \infty}\left(w(t)-w^{*}(t)\right)=0$, then, since $\hat{u}(t)$ and $u^{*}(t)$ are bounded and

$$
u^{*}(t)-\hat{u}(t)=u^{*}(t) \hat{u}(t)\left(w(t)-w^{*}(t)\right),
$$

it follows that $u^{*}(t)-\hat{u}(t) \rightarrow 0$ as $t \rightarrow \infty$. If (I) holds and $\lim _{t \rightarrow \infty}\left(w(t)-w^{*}(t)\right)$ $\neq 0$, then since $a(t) \geq a_{L}>0$ and, according to Lemma $2, \hat{v}(t) \rightarrow 0$ as $t \rightarrow \infty$, (5) implies the existence of numbers $\alpha>0$ and $t_{2} \geq t_{1}$ such that $\left|\left(w-w^{*}\right)^{\prime}(t)\right| \geq \alpha$ for all $t \geq t_{2}$. Since this contradicts the boundedness of $w(t)-w^{*}(t)$ on $\left[t_{0}, \infty\right)$, it follows that if $(\mathrm{I})$ holds, then $\lim _{t \rightarrow \infty}\left(u(t)-u^{*}(t)\right)=$ 0 .

If (II) holds, let $\tau_{n} \in\left[s_{n}, s_{n+1}\right]$ be chosen for each $n \geq 1$ such that

$$
\left|w\left(\tau_{n}\right)-w^{*}\left(\tau_{n}\right)\right|=\max _{s_{n} \leq t \leq s_{n+1}}\left|w(t)-w^{*}(t)\right|
$$

Since $\left(w-w^{*}\right)^{\prime}\left(s_{n}\right)=0$ for $n \geq 1$, it follows that $\left(w-w^{*}\right)^{\prime}\left(\tau_{n}\right)=0$ for $n \geq 1$. Therefore, by (5), $w\left(\tau_{n}\right)-w^{*}\left(\tau_{n}\right)=c\left(\tau_{n}\right) \hat{v}\left(\tau_{n}\right) w\left(\tau_{n}\right) / a\left(\tau_{n}\right)$.

Since $a(t) \geq a_{L}, w(t)$ and $c(t)$ are bounded, and $\hat{v}(t) \rightarrow 0$ as $t \rightarrow \infty$, we see that

$$
\lim _{n \rightarrow \infty}\left(w\left(\tau_{n}\right)-w^{*}\left(\tau_{n}\right)\right)=0
$$

Since $s_{n} \rightarrow \infty$ as $n \rightarrow \infty$, it follows from (6) and (7) that $w(t)-w^{*}(t) \rightarrow 0$ as $t \rightarrow \infty$. Therefore, if (II) holds we have $\lim _{t \rightarrow \infty}\left(u^{*}(t)-u(t)\right)=0$.

Since the possibilities (I) and (II) are exhaustive, the lemma is proved.

Lemma 5. Let $k_{1}>\Delta$, where $\Delta$ is a number as in Lemma 3. If $\tilde{u}(t)$ is a solution of $(\mathrm{L})$ satisfying $\tilde{u}\left(t_{0}\right)=k_{1}$, then $\tilde{u}(t)-u^{*}(t) \rightarrow 0$ as $t \rightarrow \infty$.

Proof. Since $\tilde{u}\left(t_{0}\right)>u^{*}\left(t_{0}\right)$, it follows that $\tilde{u}(t)>u^{*}(t)$ for all $t$ in $(-\infty, \infty)$. Let $w^{*}(t)=1 / u^{*}(t)$ and $\tilde{w}(t)=1 / \tilde{u}(t)$. Then, $w^{* \prime}=-a w^{*}+b$ and $\tilde{w}^{\prime}=$ $-a \tilde{w}+b$. Hence,

$$
\tilde{w}(t)-w^{*}(t)=e^{-\int_{t_{0}}^{t} a(s) d s}\left(\tilde{w}\left(t_{0}\right)-w^{*}\left(t_{0}\right)\right) \text { for } t \geq t_{0} .
$$

But,

$$
-\int_{t_{0}}^{t} a(s) d s \leq-a_{L}\left(t-t_{0}\right), \quad t \geq t_{0} .
$$

Therefore, $\tilde{w}(t)-w^{*}(t) \rightarrow 0$ as $t \rightarrow \infty$, and hence $\left(u^{*}(t)-\tilde{u}(t)\right) / u^{*}(t) \tilde{u}(t) \rightarrow 0$ as $t \rightarrow \infty$. Since $\tilde{u}(t)>u^{*}(t) \geq \delta$, we conclude that $\tilde{u}(t)-u^{*}(t) \rightarrow 0$ as $t \rightarrow \infty$.

Lemma 6. Let $k$ and $k_{1}$ be numbers as defined earlier. If $\operatorname{col}(u(t), v(t))$ is a solution of $(*)$ such that $0<u\left(t_{0}\right)<k_{1}$ and $0<v\left(t_{0}\right)<k$, then $u(t)-u^{*}(t) \rightarrow$ 0 and $v(t) \rightarrow 0$ as $t \rightarrow \infty$.

Proof. We may assume that $\varepsilon$ in Lemma 2 satisfies the inequalities $0<\varepsilon<$ $a_{M} / b_{L}<\Delta<k_{1}, \varepsilon<u\left(t_{0}\right)$, and $a_{L}-b_{M} \varepsilon-c_{M} k>0$. Recall that $\operatorname{col}(\hat{u}(t), \hat{v}(t))$ is the solution of $(*)$ such that $\hat{u}\left(t_{0}\right)=\varepsilon$ and $\hat{v}\left(t_{0}\right)=k$. We note that 
$\operatorname{col}(\tilde{u}(t), 0)$ is also a solution of $(*)$, where $\tilde{u}(t)$ is the solution of Lemma 5 satisfying the initial condition $\tilde{u}\left(t_{0}\right)=k_{1}$. Since $\hat{u}\left(t_{0}\right)<u\left(t_{0}\right)<\tilde{u}\left(t_{0}\right)$ and $\hat{v}\left(t_{0}\right)>v\left(t_{0}\right)>\tilde{v}\left(t_{0}\right)$, we have $\hat{u}(t)<u(t)<\tilde{u}(t)$ and $\hat{v}(t)>v(t)>\tilde{v}(t)$ for all $t \geq t_{0}$ (see [1]), where $\tilde{v}(t)$ denotes the second component of the solution $\operatorname{col}(\tilde{u}(t), 0)$. Since $\tilde{v}(t) \equiv 0$, and $\hat{v}(t) \rightarrow 0$ as $t \rightarrow \infty$, it follows that $v(t) \rightarrow 0$ as $t \rightarrow \infty$. Similarly, since $\hat{u}(t)-u^{*}(t)<u(t)-u^{*}(t)<\tilde{u}(t)-u^{*}(t)$, and since $\hat{u}(t)-u^{*}(t) \rightarrow 0$ and $\tilde{u}(t)-u^{*}(t) \rightarrow 0$ as $t \rightarrow \infty$, we obtain the desired result that $u(t)-u^{*}(t) \rightarrow 0$ as $t \rightarrow \infty$.

\section{MAIN ReSUlt}

We are now ready to prove our main result.

Theorem. Assume that $a(t), b(t), \ldots$, and $f(t)$ are continuous, bounded above and below by positive constants, and satisfy the inequalities in (1). If $\operatorname{col}(u(t), v(t))$ is any solution of $(*)$ such that $u\left(t_{0}\right)>0$ and $v\left(t_{0}\right)>0$ for some $t_{0}$ in $(-\infty, \infty)$, then $v(t) \rightarrow 0$ and $u(t)-u^{*}(t) \rightarrow 0$ as $t \rightarrow \infty$, where $u^{*}(t)$ is the solution of the logistic equation described in Lemma 3.

Proof. In view of the above lemmas, it suffices to show that there exists a number $t_{1} \geq t_{0}$, such that $0<u\left(t_{1}\right)<k_{1}$, and $0<v\left(t_{1}\right)<k$. To this end, suppose that $u(t) \geq k_{1}$ for $t \geq t_{0}$ (recall that $\left.k_{1}>\Delta>a_{M} / b_{L}\right)$. Then $u^{\prime}(t)=u(t)[a(t)-b(t) u(t)-c(t) v(t)] \leq u(t)\left[a(t)-b(t) k_{1}\right]<0$. Hence, $u^{\prime}(t) / u(t) \leq a(t)-b(t) k_{1}<a_{M}-b_{L} k_{1}<0$. But this implies that $\ln u(t) \rightarrow-\infty$, and hence $u(t) \rightarrow 0$ as $t \rightarrow \infty$, which is a contradiction. This shows that there exists a number $\bar{t}_{1} \geq t_{0}$ such that $u\left(\bar{t}_{1}\right)<k_{1}$. Similarly, there exists a number $\bar{t}_{2} \geq t_{0}$, such that $v\left(\bar{t}_{2}\right)<k$. Let $t_{1}=\max \left(\bar{t}_{1}, \bar{t}_{2}\right)$, and the proof is complete.

\section{REFERENCES}

1. S. Ahmad, Convergence and ultimate bounds of solutions of the nonautonomous VolterraLotka competition equations, J. Math. Anal. Appl. 127 (1987), 377-387.

2. __ On almost periodic solutions of the competing species problems, Proc. Amer. Math. Soc. 102 (1988), 855-861.

3. S. Ahmad and A. C. Lazer, Asymptotic behavior of solutions of periodic competition diffusion systems, Nonlinear Anal. 13 (1989), 263-283.

4. C. Alvarez and A. C. Lazer, An application of topological degree to the periodic competing species problem, J. Austral. Math. Soc. Ser. B 28 (1986), 202-219.

5. B. D. Coleman, Nonautonomous logistic equations as models of the adjustment of populations to environmental change, Math. Biosciences 45 (1979), 159-173.

6. K. Gopalsamy, Exchange of equlibria in two species Lotka-Volterra competition models, J. Austral. Math. Soc. Ser. B 24 (1982), 160-177.

7. J. M. Smith, Mathematical ideas in biology, Cambridge Univ. Press, London, 1968.

8. L. Zhou and C. V. Pao, Asymptotic behavior of a competition-diffusion system in population dynamics, Nonlinear Anal. 6 (1982), 1163-1184.

Division of Mathematics, Computer Science and Statistics, The University of Texas at San Antonio, 6900 North Loop West, San Antonio, Texas 78249

E-mail address: ahmad@ringer.c.s.utsa.edu 